Recepción: 20 / 04 / 2017

Aceptación: 20 / 05 / 2017

Publicación: 15 / 07 / 2017

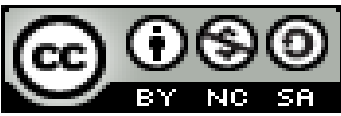

Ciencias Médicas

Artículo Científico

\title{
Propuesta de un programa educativo a pacientes y familiares para prevenir la deshidratación severa por enfermedad diarreica aguda a niños menores de 5 años
}

\section{Proposal of a patient and family educational program to prevent severe dehydration by acute diarrhea disease for children under 5 years old}

\section{Proposta de um programa educacional para pacientes e familiares para prevenir a desidratação severa por doença diarréica aguda para crianças com menos de 5 anos de idade}

\author{
Maritza A. Borja-Santillán I \\ Marco A. Calle-Gómez III \\ maritza.borjas@ug.edu.ec \\ marco.calleg@ug.edu.ec \\ Janet P. Emén-Sánchez II \\ Ofelia Jaime-Game ${ }^{\text {IV }}$ \\ janet.emens@ug.edu.ec \\ ofelia.jaimeg@ug.edu.ec
}

Correspondencia: maritza.borjas@ug.edu.ec

\footnotetext{
I. Magister en Emergencias Médicas; Magister en Gerencia de Salud para el Desarrollo Local; Especialista en Gerencia y Planificación Estratégica en Salud; Diploma Superior en Desarrollo Local y Salud; Profesora de Segunda Enseñanza Especialización Lengua Inglesa y Lingüística; Licenciada en Ciencias de la Educación mención Lengua Inglesa y Lingüística; Medico; Profesora Titular Auxiliar de la Facultad de Ciencias; Médicas, Escuela de Medicina, Universidad de Guayaquil, Guayaquil, Ecuador.

II. Master Universitario en Nutrición Humana y Dietética Aplicada; Especialista en Medicina del Deporte; Medica; Profesora Titular Auxiliar de la Facultad de Ciencias Médicas; Escuela de Medicina; Universidad de Guayaquil, Guayaquil, Ecuador.

III. Especialista en Cirugía, Especialista en Anestesiología, Doctor En Medicina y Cirugía, Abogado de los Tribunales y Juzgados de la Republica, Diploma Superior en Docencia Universitaria, Universidad deGuayaquil, Guayaquil, Ecuador.

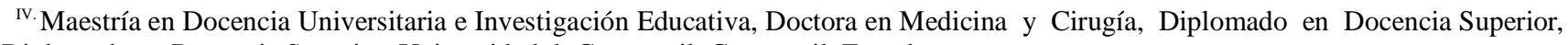
Diplomado en Docencia Superior, Universidad de Guayaquil, Guayaquil, Ecuador.
} 


\section{Resumen}

La deshidratación aguda es una alteración del balance hidroelectrolítico del organismo ocasionada por disminución del aporte de líquidos, aumento en las pérdidas corporales o ambas cosas simultáneamente, pudiendo generar un compromiso de las funciones corporales ${ }^{1,}{ }^{2}$. Por lo cual se considera como el problema central del presente estudio el alto índice de deshidratación por enfermedad diarreica aguda en niños menores de 5 años.

La investigación plantea como objetivo general y resultado esperado, "proponer un programa educativo preventivo para la deshidratación severa por enfermedad diarreica aguda (EDA), en niños menores de 5 años", con el fin de disminuir la prevalencia de deshidratación en este grupo etario lo cual contribuiría a disminuir la mortalidad por EDA. Determinando la prevalencia, factores de riesgo, grupos etarios más afectados, grados de deshidratación, y conducta a seguir por el personal de salud del Centro de salud Durán, siendo posible identificar el alcance del programa educativopreventivo que se propone. De esta manera se pretende incidir sobre la disminución de casos de deshidratación por EDA mediante la prevención y la incidencia en políticas institucionales.

En relación al diseño de investigación es de tipo no experimental, de alcance descriptivo, retrospectivo. La técnica de recolección de datos se realizó a través de revisión de historias clínicas. Para el análisis de datos se empleó una tabla de doble entrada según las variables propuestas, las cuales permitieron la verificación de la hipótesis a la vez que ayudaron a conocer la realidad de este problema de salud. El resultado de esta investigación fue puesto en consideración de los Directivos del Centro de Salud para que sea considerado como lineamiento de planes, programas y proyectos, para la prevención de deshidratación por EDA y su incorporación dentro de las políticas institucionales.

Palabras clave: Deshidratación; enfermedad diarreica aguda; niños menores de 5 años; prevención; programa; evaluación, atención médica. 


\begin{abstract}
Acute dehydration is a disruption of electrolyte balance in the body caused by decreased fluid intake, weight losses increase in body or both simultaneously, this can potentially lead to a difficulty of bodily functions ${ }^{1,2}$. Hence, it is considered as the central problem of this study, the high rate of dehydration from acute diarrhea in children under five years old.
\end{abstract}

This Research presents as a general objective and expected outcome "to propose a preventive educational program for severe dehydration for acute diarrhea disease (ADD) in children under five years old", in order to reduce the prevalence of dehydration in this age group and it would contribute to decrease the mortality by ADD. By determining the prevalence, risk factors, most affected age groups, degree of dehydration, and procedure followed by the health personnel at health center "Durán", being possible to identify the scope of the proposed educational-preventive program.

In this way, it is intended to influence the reduction of cases of ADD dehydration through prevention and incidence in institutional policies. In relation to research design, it's a nonexperimental type, descriptive and retrospective scope. The technique of data collection was done through review of medical records. For data analysis there will be used a two-way table according to the proposed variables, which allowed the verification of the hypothesis at the same time that helped to know the reality of this health problem. The result of this research was to put into consideration by the Directors of the Health Center to be considered as a guideline of plans, programs and projects for the prevention of ADD dehydration and its incorporation into institutional policies.

Key words: Acute pain; analgesic; ketamine; visual analog scale; intranasal. 


\section{Resumo}

A desidratação aguda é uma ruptura do equilíbrio eletrolítico no corpo causada pela diminuição da ingestão de líquidos, as perdas de peso aumentam no corpo ou em ambos simultaneamente, o que pode levar a uma dificuldade de funções corporais1,2. Por isso, é considerado o problema central deste estudo, a alta taxa de desidratação da diarréia aguda em crianças com menos de cinco anos de idade.

Esta pesquisa apresenta como objetivo geral e resultado esperado "propor um programa educacional preventivo para desidratação severa para doença de diarréia aguda (ADD) em crianças menores de cinco anos", a fim de reduzir a prevalência de desidratação nesta faixa etária e Contribuem para diminuir a mortalidade por ADD. Ao determinar a prevalência, os fatores de risco, as faixas etárias mais afetadas, o grau de desidratação e o procedimento seguido pelo pessoal de saúde no centro de saúde "Durán", sendo possível identificar o escopo da educação educacional proposta, Programa preventivo.

Desta forma, pretende influenciar a redução de casos de desidratação ADD através da prevenção e incidência nas políticas institucionais. Em relação ao projeto de pesquisa, é um tipo não experimental, um escopo descritivo e retrospectivo. A técnica de coleta de dados foi feita através da revisão de registros médicos. Para a análise de dados, será utilizada uma tabela de duas vias de acordo com as variáveis propostas, o que permitiu a verificação da hipótese ao mesmo tempo que ajudou a conhecer a realidade desse problema de saúde. O resultado desta pesquisa foi levar em consideração pelos Diretores do Centro de Saúde para ser considerado como uma diretriz de planos, programas e projetos para a prevenção da desidratação ADD e sua incorporação em políticas institucionais.

Palavras chave: Dor aguda; analgésico; Ketamina; Escala analógica visual; Intranasal. 


\section{Introducción.}

Las enfermedades diarreicas son la segunda mayor causa de muerte de niños menores de cinco años a nivel mundial, siendo una de sus graves consecuencias la deshidratación ${ }^{3}$. Las enfermedades diarreicas matan a 525.000 niños menores de cinco años cada año; en la actualidad, diversas enfermedades como las causadas por infecciones bacterianas septicémicas, están relacionadas con las muertes por diarrea ${ }^{4}$. Una proporción significativa de las enfermedades diarreicas se puede prevenir mediante el acceso al agua potable y a servicios adecuados de saneamiento e higiene. En todo el mundo se producen alrededor de 1.5 millones de casos de enfermedades diarreicas cada año, siendo una de las principales causas de malnutrición de niños menores de cinco años $5,6,7$.

Las enfermedades diarreicas agudas continúan siendo una causa importante de morbimortalidad en muchos países del mundo, sobre todo en los países del Tercer Mundo; por ejemplo, en América Latina se presentan 250 millones de casos por año y mueren 3,2 millones de niños menores de 5 años ${ }^{8,9}$, convirtiéndose en una de las causas más importantes de mortalidad infantil en los países en vías de desarrollo y uno de los principales motivos de ingreso y atención hospitalaria. Las complicaciones por enfermedades diarreicas no son sucesos inevitables, sino que la inmensa mayoría pueden prevenirse con reorientación, educación, fortalecimiento de los cuidados que deben tenerse con los niños; esta es la clave fundamental para evitar no solamente las complicaciones, sino además las reinfecciones ${ }^{5,6}$. La enfermedad diarreica tiene una alta incidencia en el Ecuador por ser este un país multicultural, multiétnico y en donde la población infantil es alta; sin embargo, no constituye esta afectación un problema de mortalidad, pero sí de morbilidad ${ }^{10}$. A pesar de mantener una baja tasa de mortalidad por enfermedades diarreicas, en el Ecuador aún se identifican problemas, y dentro de ellas la persistencia de costumbres y hábitos inadecuados que 
favorecen la aparición de complicaciones. Las costumbres persistentes más frecuentes son: las restricciones dietéticas, el uso de medicamentos antimicrobianos y antidiarreicos, prácticas oscurantistas como la cura del empacho o la administración de remedios caseros. Otros hábitos inadecuados son la demora en asistir a la consulta médica en busca de orientación y la atribución a causas oscuras y no demostradas, como la dentición y el resfriado ${ }^{7}$.

Por tal motivo, el objetivo de este estudio consiste en proponer un programa educativo preventivo para la deshidratación severa por enfermedad diarreica aguda en niños menores de 5 años, dirigido a sus familiares. La hipótesis planteada para este estudio fue que diseñando y difundiendo un programa educativo-preventivo para la deshidratación severa por EDA se logrará disminuir la morbi-mortalidad de los niños menores de 5 años que acuden al centro de salud Durán.

\section{Materiales y métodos.}

La presente investigación tuvo un enfoque cuantitativo, de alcance descriptivo y de tipo no experimental, retrospectivo.

La investigación se efectuó en el Centro de Salud Durán que se encuentra ubicado en el cantón Durán de la provincia del Guayas. El periodo de investigación comprendió de enero a junio del 2013 con los casos de EDA registrados durante el mismo. La muestra consistió en un total de 285 niños menores de 5 años de edad y el personal de atención en salud que fueron 7 médicos.

Para este estudio fue de gran importancia la información obtenida de las historias clínicas de los pacientes y del personal médico, así como la información proporcionada por el departamento de estadística del Centro de Salud Durán. A partir de ello se obtuvo información referente al grado de deshidratación de los pacientes, el tipo de hidratación y de tratamiento recibidos en el centro de salud, así como la necesidad de derivación de los casos a unidades médicas de mayor complejidad. 
Se determinó, además, por medio de encuestas, la existencia y ejecución de programas educativo-preventivos para el manejo de la EDA a familiares y personal de salud y su frecuencia, existencia de protocolos de manejo de la EDA y su fácil acceso y conocimiento por parte del personal de salud, la presencia de una cantidad suficiente de personal para la atención de los casos de emergencia, así como la cantidad necesaria de insumos y el espacio adecuado para la atención de la EDA.

Se obtuvieron también otros datos importantes como la edad y sexo de los pacientes, factores de riesgo asociados a la patología, esquema de vacunación del niño y como factores de riesgo asociados a la EDA se indagó la persona a cargo del cuidado del niño, nivel educativo de los padres y/o cuidador, tipo de alimentación del niño, así como aspectos de salubridad referentes al agua y servicios sanitarios del domicilio.

\section{Resultados.}

La prevalencia de atención de pacientes con EDA en el centro de salud Durán fue de un 22\% en relación a la totalidad de la población atendida durante el primer semestre del 2013.

\begin{tabular}{ccc}
\hline REFERENCIA & CANTIDAD & PORCENTAJE \\
\hline Pacientes con EDA & 993 & $22 \%$ \\
Pacientes sin EDA & 3535 & $78 \%$ \\
Población total & 4528 & \\
\hline
\end{tabular}

\section{Cuadro $N^{\circ}$ 1.-Pacientes con EDA en el primer semestre 2013 en el centro de salud Durán}

La población de pacientes estuvo representada por 129 varones (45\%) y 156 mujeres (55\%), en edades comprendidas por 96 niños menores de un año (34\%), seguido de 136 niños entre uno y tres años de edad $(47,7 \%)$ y finalmente 53 niños entre tres y cinco años $(18,6 \%)$. 


\begin{tabular}{ccc}
\hline REFERENCIA & CANTIDAD & PORCENTAJE \\
Menores de 1 año & 96 & $34 \%$ \\
1 a 3 años & 136 & $47,7 \%$ \\
3 a 5 años & 53 & $18,6 \%$
\end{tabular}

\section{Cuadro $N^{\circ}$ 2.- Grupo etario pacientes con EDA}

En cuanto a la prevalencia de deshidratación en los pacientes que conformaron la muestra de esta investigación, encontramos un 48\% en la deshidratación leve que corresponde a 138 niños; 96 pacientes se ubicaron en la categoría de deshidratación moderada, lo cual representó el 34\% de la muestra, y finalmente, en la deshidratación severa se encontraron 51 casos que correspondieron al $18 \%$.

\begin{tabular}{ccc}
\hline REFERENCIA & CANTIDAD & PORCENTAJE \\
Leve & 138 & $48 \%$ \\
Moderado & 96 & $34 \%$ \\
Severo & 51 & $18 \%$
\end{tabular}

\section{Cuadro $N^{\circ}$ 3.- Nivel de deshidratación por EDA}

En cuanto a los tratamientos aplicados a la muestra, encontramos que el 68\% (195 niños) recibió hidratación oral, el 30\% (85 niños) recibió hidratación parenteral y finalmente el 2\% (5 niños) no tuvo tratamiento por hidratación. 


\begin{tabular}{c|cc} 
REFERENCIA & CANTIDAD & PORCENTAJE \\
\hline Hidratación Oral & 195 & $68 \%$ \\
Hidratación Parenteral & 85 & $30 \%$ \\
Sin tratamiento de hidratación & 5 & $2 \%$
\end{tabular}

\section{Cuadro $N^{\circ}$ 4.- Tipo de hidratación}

En lo que respecta al tratamiento medicamentoso, 147 pacientes (52\%) recibieron antibioticoterapia, 20 pacientes (7\%) recibieron antimicóticos, la misma cantidad (7\%) no recibió medicación alguna, 98 pacientes (34\%) recibieron antiparasitarios y, finalmente, el uso de antivirales no se requirió en ninguno de los casos.

Del total de pacientes estudiados, el 20\% (57 niños) tuvo necesidad de ser transferido a una unidad de salud de mayor nivel de complejidad y el $80 \%$ (228 pacientes) no fue transferido.

Entre los factores de riesgo para la enfermedad, se encontró que la persona que está al cuidado del niño es mayoritariamente la madre (52\%), seguida de otras personas (40\%), la presencia del padre se dio en un 5\% y finalmente un $8 \%$ no registró datos. El nivel educativo de los padres y/o cuidadores estuvo representado por un 54\% (154 adultos) con educación primaria, le sigue el 31\% (88 personas) sin ningún tipo de educación, el estudio secundario recayó en el 8\% (23 adultos) y finalmente el 3\% (8 adultos) no registra datos en esta categoría.

En cuanto al factor de riesgo asociado a la vacunación correspondiente a la edad del menor, se registró que el 79\% sí recibió vacunación acorde a su edad, el 18\% no recibió la vacunación adecuada a la edad y el $4 \%$ no expresa información a este respecto. 
En lo relacionado al tipo de alimentación, el 16\% de los pacientes recibió alimentación de leche materna, el 17\% recibió leche de fórmula, el 48\% recibió ambas y finalmente el $19 \%$ refirió haber tenido otro tipo de alimentación.

En lo que se refiere a características de salubridad, el 72\% refirió tener acceso a agua potable, el $21 \%$ adquiere agua por tanquero y el $8 \%$ adquiere agua de pozo. La eliminación de excretas en un $28 \%$ se hace por letrina/pozo séptico y el $72 \%$ por alcantarillado.

\begin{tabular}{c|cc} 
REFERENCIA & CANTIDAD & PORCENTAJE \\
Potable & 204 & $72 \%$ \\
Tanquero & 59 & $21 \%$ \\
Pozo & 22 & $8 \%$
\end{tabular}

Cuadro $N^{\circ}$ 5.- Tipo de salubridad del agua

\begin{tabular}{c|cc} 
REFERENCIA & CANTIDAD & PORCENTAJE \\
Letrina / Pozo Séptico & 81 & $28 \%$ \\
Alcantarillado & 204 & $72 \%$
\end{tabular}

\section{Cuadro $N^{\circ}$ 6.- Tipo de sistema de saneamiento}

Como acciones realizadas en el manejo de la EDA, se conoció que no existe ni se aplica un programa educativo-preventivo para padres y familiares en el centro de salud, lo cual fue referido en un $86 \%$ por el personal de salud, mientras que el $14 \%$ refirió que sí existe. Adicional a esto, se indagó sobre la existencia de un protocolo de atención para la EDA, obteniendo como resultado que el 57\% del personal refirió que sí existía, el 29\% refirió no haber y el 14\% expresó desconocer; de 
igual manera, el 29\% expresó que el protocolo era de fácil acceso, el 57\% manifestó que era de difícil acceso y finalmente el 14\% expresó desconocer su accesibilidad.

Con respecto a la difusión de algún programa educativo-preventivo de la EDA en la comunidad correspondiente al centro de salud investigado, el 29\% de los médicos expresó que sí existe difusión de estrategias preventivas, mientras que el $71 \%$ expresó que no existe. De igual manera, el 14\% expresó que esta difusión tiene lugar una vez al año, otro 14\% expresó que la difusión tiene una frecuencia de dos veces al año, para finalizar, el 71\% se mantuvo en la respuesta de la no existencia de difusión de ningún programa educativo-preventivo.

Se indagó también acerca de la existencia de capacitaciones al personal de salud para el manejo de la EDA; como resultado se obtuvo que el 57\% de los médicos manifestó que sí existía capacitación al personal mientras que el $43 \%$ expresó que no. Estas capacitaciones tienen lugar una vez al año según el $57 \%$ de los médicos, el $43 \%$ se mantuvo en la respuesta de la no existencia de capacitaciones al personal. Por otro lado, el $71 \%$ refirió que la cantidad de personal para la atención de emergencias médicas no era suficiente, mientras que el 29\% refirió que sí era suficiente.

En lo que respecta al área física para la atención de deshidratación severa en niños menores de 5 años, el $100 \%$ de los médicos manifestó que no existía un área física adecuada para la atención de estos casos. En cuanto a los insumos para la atención de esta patología, el 57\% expresó que sí contaban con insumos necesarios y el $43 \%$ expresó que no.

\section{Discusión.}

Las enfermedades diarreicas, según la OMS, constituyen la segunda mayor causa de muerte en niños menores de cinco años, con un promedio de 525.000 muertes por año. Las acciones preventivas como el correcto lavado de manos, el acceso a agua potable, adecuados sistemas de 
saneamiento, así como la correcta hidratación al menor, pueden disminuir el riesgo de contagio y muertes.

Los resultados obtenidos en este estudio corroboraron la necesidad de aplicar un programa educativo-preventivo para el manejo adecuado de la EDA en niños menores de 5 años, dirigido a sus familiares e, incluso, al personal de atención en emergencias. Dado que la presencia de la patología en el centro de salud Durán tuvo una prevalencia del 22\%, durante el 1er semestre del 2013, es palpable la necesidad de implementar este tipo de programas para la comunidad.

La EDA conlleva necesariamente a la deshidratación, lo cual se corroboró en este estudio puesto que todos los pacientes estudiados presentaron diferentes niveles de deshidratación. Aunque el centro de salud refirió tener los insumos necesarios para la atención de esta patología, no existe el personal suficiente para la atención en emergencias, así como tampoco existe un programa de capacitación para los familiares, en el cual puedan apoyarse para la prevención y tratamiento oportunos y adecuados de la EDA.

El riesgo de presentar deshidratación se asoció con las condiciones socioeconómicas desfavorables; la dificultad de acceder al consumo de agua potable y sistemas de saneamiento adecuados, así como el bajo nivel educativo de la madre o de los cuidadores del niño, constituyeron los factores de riesgo más importantes encontrados en este estudio.

Probablemente la poca atención que tiene esta patología, lo cual está reflejado en la escasez de personal de atención, conocimiento inadecuado del manejo de la EDA, entre otros aspectos investigados en este estudio, sea producto de que nuestra sociedad haya normalizado esta enfermedad como algo cotidiano que no requiere de mayor atención, aumentando el riesgo de complicaciones en el desarrollo del niño e incluso la muerte. 
Consideramos que este este estudio permite reconocer la necesidad de intervenir con la difusión de información referente a la EDA a nivel provincial y de país, enfatizando en campañas preventivas desde los actores involucrados en diversas áreas de atención infantil como el Ministerio de Salud Pública (MSP), Ministerio de Inclusión Económica y Social (MIES) y Ministerio de Educación (Mineduc), con la finalidad de garantizar el desarrollo saludable en la primera infancia en nuestro país. Para esto es recomendable que se realice un próximo estudio haciendo una comparación entre todos los centros de salud del área.

\section{Conclusión.}

Con lo expuesto con anterioridad se concluye que existe una imperante necesidad de instaurar como programaciones anuales campañas de prevención de enfermedades con el fin de disminuir la morbilidad de la población en espera de no llegar a cuantificar mortalidad.

Disminuir los factores de riesgo, la preparación de personal de manera continua, así como capacitar a la población, desempeña un papel importante en la prevención de la deshidratación por enfermedad diarreica aguda.

El presente estudio propone, por tanto, un programa educativo-preventivo dirigido a familiares de pacientes menores de cinco años de edad con EDA, que posibilita el tratamiento adecuado y la prevención de la patología, como aporte a acciones implementadas en favor del desarrollo infantil.

\section{Bibliografía.}

1) Pérez-Schael I. Deshidratación. Investigación clínica pediátrica. 2011; 49(4):508.

2) Stampone J. Conocimientos y aplicaciónn de la fluidoterapia en pacientes deshidratados por parte del personal médico en el departamento de Pediatría. PortMed. [Internet]. 2010 [citado 13 mayo 2015]. Disponible en: http://www.salamandra.edu.co/fileadmin/imagenes/imagenes_articulos/Fluidoter apia.pdf

3) MedlinePlus [Internet]. Estados Unidos: MedlinePlus. [citado 10 junio 2015]. Deshidratación [aproximadamente 2 pantallas]. Disponible en: https://medlineplus.gov/spanish/ency/article/000982.htm 
4) Who.int [Internet]. Organización Mundial de la Salud, 2017 [citado 13 mayo 2015]. Enfermedades diarreicas $\quad$ [aproximadamente 2 pantallas]. http://www.who.int/mediacentre/factsheets/fs330/es/

5) OPS. Uso racional de medicamentos en el manejo de la diarrea aguda en niños. Serie Paltex No23. Estados Unidos: Organización Panamericana de la Salud; 1991.

6) OPS. Manual de tratamiento de la diarrea. Serie Paltex Nº13. Estados Unidos: Organización Panamericana de la Salud. 1987.

7) Cuevas R, Rodríguez K, Muñiz V, Castro V, Maturell M. Enfermedad diarreica aguda en niños guatemaltecos menores de 5 años. Medisan [Internet]. 2014 [citado 23 mayo 2015]; 8(11). Disponible en: http://scielo.sld.cu/scielo.php?script=sci_arttext\&pid=S1029-30192014001100005

8) Gil FJ, Martínez D, Nadal I. Deshidratación aguda. Pediat [Internet]. [citado 23 mayo 2015]. Disponible en: $\quad$ http://www.cfnavarra.es/salud/PUBLICACIONES/Libro\%20electronico\%20de $\% 20$ temas\%20de\%20Urgencia/21.Pediatricas/Deshidratacion\%20pediatria.pdf

9) Álvarez-Calatayud G, Taboada L, Rivas A. Deshidratación: etiología, diagnóstico y tratamiento. An Pediatr Contin [Internet]. 2006 [citado 23 mayo 2015]; 4(5). Disponible en: http://www.apcontinuada.com/es/deshidratacion-etiologiadiagnostico-tratamiento/articulo/80000209/ 